\section{Set-Membership Filtering with State Constraints}

In this paper, the problem of set-membership filtering is considered for discrete-time systems with equality and inequality constraints between their state variables. We formulate the problem of set-membership filtering as finding the set of estimates that belong to an ellipsoid. A centre and a shape matrix of the ellipsoid are used to describe the set of estimates and the solution to the set of estimates is obtained in terms of matrix inequality. Unknown but bounded process and measurement noises are handled under the inequality constraints by using S-procedure. We apply Finsler's Lemma to project the set of estimates onto the constrained surface. A recursive algorithm is developed for computing the ellipsoid that guarantees to contain the true state under the state constraints, which is easily implemented by semi-definite programming via interior-point approach. A vehicle tracking example is provided to demonstrate the effectiveness of the proposed set-membership filtering with state equality constraints.

\section{INTRODUCTION}

The topic of set-membership filtering has attracted a growing research interest, since it is based only on the knowledge of the hard bounds of the process and measurement noises $[3,6,8,10$, 13, 14, 16-21, 29]. The idea of set-membership filtering is to provide all possible state estimates that are characterised by the set of state estimates consistent with both the observations received and the unknown but bounded process and measurement noises $[3,10,20]$. The set-membership filtering can find a region in the state-space that guarantees to contain the unknown true state vector [13]. Hence the set-membership filtering problem aims to find the smallest characterisation of the feasible set of the states, rather than providing the most possible states under some optimality criteria, for example, Kalman filtering $[2,27,33,34,36,38]$ and $H_{\infty}$ filtering $[28,30,32]$. Set-membership filtering is also called set-value filtering as the actual estimate is a set in state space rather than a single vector [14], [17], [21].

Manuscript received November 16, 2007; revised May 21 and August 17, 2008; released for publication September 23, 2008.

IEEE Log No. T-AES/45/4/935115.

Refereeing of this contribution was handled by B. La Scala.

This work was supported by the Engineering and Physical Science Research Council (EPSRC) of the U.K. under Grant EP/C007654/1, and the National Nature Science Foundation of China under Grant 60874059 .

0018-9251/09/\$26.00 (C) 2009 IEEE
Set-membership filtering problem was first considered by Witsenhausen [29]. The set of all possible values of the states compatible with observation of outputs is completely characterised by their support functions. An efficient algorithm with certain computational advantages was provided by Schweppe [20] for the set-membership filtering problem under the energy-type constraint. The solution to a set-membership filtering problem with the individual instantaneous constraints was determined by describing a bounding ellipsoid to the set of possible states [3]. The resulting filter is similar to that proposed by Schweppe [20], but it has an important advantage that the gain matrix does not depend on the particular output observations and is therefore precomputable. Recently, attempts have been made to deal with the set-membership filtering problems for uncertain systems. For example, a combinational ellipsoidal bounded uncertain system was considered in [16]. The sum quadratic uncertain systems have been studied in [17]-[19]. For systems with both bounded noise and parametric uncertainty, a technique-based semi-definite optimization method has been proposed in [8] to handle several inequalities. It has led to a simple and neat algorithm. We adopt this technique in this paper.

However, in practical applications such as vehicle tracking, there are some hard constraints on the vehicle position when the vehicle is travelling on a known road (straight line or curve). Such tracking problem can be regarded as a filtering problem incorporating a state constraint with the road network information from digital maps [11, 22, 37]. This paper intends to study the set-membership filtering problem incorporating state constraints. The filtering problems with state constraints have been studied within the Kalman filter framework [9, 24, 31]. There have been several approaches to address this problem, which can be classified into augmented measurement and projection approaches. The augmented measurement approach is to treat the state constraints as additional fictitious or pseudo measurements in perfect forms (i.e., no measurement noise) $[1,5,26]$. This approach is simple and intuitive, but the incorporation of state constraints as perfect measurements brings the possibility of numerical problems and increases the dimension of the problem [24]. The projection approach is first to obtain an unconstrained Kalman filter solution and then project the unconstrained state estimate onto the constrained surface $[9,23$, $24,31]$. The approach overcomes the numerical and dimensional problems. The key point of this approach is to find an appropriate projection method.

In this paper, we address the filtering problems with state constraints within the set-membership filter framework. Both state equality and inequality constraints are considered. We first adopt the $\mathrm{S}$-procedure method to transfer all inequalities into 
one inequality and then obtain the solution to the unconstrained set-membership filtering problem. We finally apply Finsler's Lemma to project the unconstrained solution onto the constrained surface. The constrained set-membership filter is designed by solving a linear matrix inequality (LMI). A recursive algorithm is developed for computing the ellipsoid that guarantees to contain the true state under the state constraints.

The remainder of this paper is organized as follows. The set-membership filtering problem with state constraints is formulated for discrete-time systems in Section II. A set-membership filter with state equality constraints is designed for determining a state estimation ellipsoid where the true state resides in Section III. The results of Section III are extended to study the set-membership filtering problem with state inequality constraints in Section IV. A vehicle tracking example is provided in Section V to demonstrate the effectiveness of our method. Conclusions are drawn in Section VI.

Notation: The notation $X \geq Y$ (respectively, $X>Y$ ) where $X$ and $Y$ are symmetric matrices, means that $X-Y$ is positive semi-definite (respectively, positive definite). The superscript $T$ stands for matrix transposition. The notation $\operatorname{tr}(P)$ denotes the trace of $P$.

\section{PROBLEM FORMULATION}

Consider the following discrete time-varying system:

$$
\begin{aligned}
x_{k+1} & =A_{k} x_{k}+F_{k} u_{k}+B_{k} w_{k} \\
y_{k} & =C_{k} x_{k}+D_{k} v_{k}
\end{aligned}
$$

where $x_{k} \in \mathbb{R}^{n}$ is the system state, $u_{k} \in \mathbb{R}^{l}$ is the known deterministic input, $y_{k} \in \mathbb{R}^{m}$ is the measurement output, $w_{k} \in \mathbb{R}^{r}$ is the process noise, and $v_{k} \in \mathbb{R}^{p}$ is the measurement noise. $A_{k}, B_{k}, C_{k}, D_{k}$, and $F_{k}$ are known time-varying matrices with appropriate dimensions.

In addition to the dynamic system (1), there exist the state constraints. Two constraints are considered in this paper. One is the state equality constraint in the form of

$$
S_{k} x_{k}=s_{k}
$$

where $S_{k}$ is a known time-varying matrix, $s_{k}$ is a known time-varying vector, and the number of rows in $S_{k}$ is the number of constraints, which is assumed to be less than the number of states. The other is the state inequality constraint which satisfies the following inequality:

$$
x_{k}^{T} J_{k} x_{k}<a_{k}
$$

where $J_{k}$ is a known time-varying positive semi-definite matrix, and $a_{k}$ is a known time-varying positive scalar.
REMARK 1 The state constraints exist in many physical systems. For example, the travelling vehicle makes use of the road geometric information as a constraint, such as, straight line or curve $[11,15,22]$. The vision-based tracking systems require unit quaternions as constraint $[7,9,15]$. The coordinated turn model for an aircraft assumes that the acceleration vector is orthogonal to the velocity vector as a constraint $[1,26]$.

It is assumed that process and measurement noises are confined to specified ellipsoidal sets:

$$
\begin{aligned}
\mathcal{W}_{k} & =\left\{w_{k}: w_{k}^{T} Q_{k}^{-1} w_{k} \leq 1\right\} \\
\mathcal{V}_{k} & =\left\{v_{k}: v_{k}^{T} R_{k}^{-1} v_{k} \leq 1\right\}
\end{aligned}
$$

where $Q_{k}=Q_{k}^{T}>0$ and $R_{k}=R_{k}^{T}>0$ are known matrices with compatible dimensions. The initial state $x_{0}$ belongs to a given ellipsoid:

$$
\left(x_{0}-\hat{x}_{0}\right)^{T} P_{0}^{-1}\left(x_{0}-\hat{x}_{0}\right) \leq 1
$$

where $\hat{x}_{0}$ is an estimate of $x_{0}$ which is assumed to be given, and $P_{0}=P_{0}^{T}>0$ is a known matrix.

In this paper, a filter based on the current measurement is considered for the system (1)-(2) subject to the constraint (3) or (4), which is of the form:

$$
\hat{x}_{k+1}=G_{k} \hat{x}_{k}+F_{k} u_{k}+L_{k} y_{k+1}
$$

where $\hat{x}_{k} \in \mathbb{R}^{n}$ is the state estimate of $x_{k}$. $G_{k}$ and $L_{k}$ are the filter parameters to be determined.

Our aim is to determine an ellipsoid for the state $x_{k}$, given the measurement information $y_{k}$ at the time instant $k$ for the process noise $w_{k} \in \mathcal{W}_{k}$ and the measurement noise $v_{k} \in \mathcal{V}_{k}$ subject to the state constraints (3) or (4). In other words, we look for $P_{k}$ and $\hat{x}_{k}$ such that

$$
\left(x_{k}-\hat{x}_{k}\right)^{T} P_{k}^{-1}\left(x_{k}-\hat{x}_{k}\right) \leq 1
$$

subject to $w_{k} \in \mathcal{W}_{k}, v_{k} \in \mathcal{V}_{k}$, and (3) or (4).

The above filtering problem is referred to as the set-membership filtering problem with state constraints.

\section{SET-MEMBERSHIP FILTER DESIGN WITH STATE EQUALITY CONSTRAINTS}

In this section, we consider the set-membership filter design problem with state equality constraint (3). In order to develop the filter, we need the following three useful lemmas:

LEMMA 1 (S-procedure) [4, 25] Let $Y_{0}(\eta), Y_{1}(\eta)$, $\ldots, Y_{p}(\eta)$ be quadratic functions of $\eta \in \mathbb{R}^{n}$

$$
Y_{i}(\eta)=\eta^{T} T_{i} \eta, \quad i=0,1, \ldots, p
$$

with $T_{i}=T_{i}^{T}$. Then, the implication

$$
Y_{1}(\eta) \leq 0, \ldots, Y_{p}(\eta) \leq 0 \Longrightarrow Y_{0}(\eta) \leq 0
$$


holds if there exist $\tau_{1}, \ldots, \tau_{p}>0$ such that

$$
T_{0}-\sum_{i=1}^{p} \tau_{i} T_{i} \leq 0
$$

LEMMA 2 (Schur Complements) [4] Given constant matrices $L_{1}, L_{2}, L_{3}$ where $L_{1}=L_{1}^{T}$ and $L_{2}=L_{2}^{T}<0$, then

$$
L_{1}-L_{3}^{T} L_{2}^{-1} L_{3} \leq 0
$$

if and only if

$$
\left[\begin{array}{ll}
L_{1} & L_{3}^{T} \\
L_{3} & L_{2}
\end{array}\right] \leq 0
$$

or equivalently

$$
\left[\begin{array}{ll}
L_{2} & L_{3} \\
L_{3}^{T} & L_{1}
\end{array}\right] \leq 0
$$

LEMma 3 (Finsler's Lemma) [25, 35] Let $x \in \mathbb{R}^{n}$, $P=P^{T} \in \mathbb{R}^{n \times n}$, and $M \in \mathbb{R}^{m \times n}$ such that $\operatorname{rank}(M)=r<$ $n$. The following statements are equivalent:

1) $x^{T} P x \leq 0, \forall M x=0, x \neq 0$.

2) $\left(M^{\perp}\right)^{T} P M^{\perp} \leq 0$.

3) $\exists \mu \in \mathbb{R}: P-\mu M^{T} M \leq 0$.

4) $\exists N \in \mathbb{R}^{m \times n}: P+N^{T} M+M^{T} N \leq 0$.

\section{REMARK 2}

1) $M^{\perp}$ is a basis for the null space of $M$. That is, all $x \neq 0$ such that $M x=0$ is generated by some $z \neq 0$ in the form $x=M^{\perp} z$.

2) $N=-(\mu / 2) M^{T}$ is a solution for 4$)$ in Lemma 3 .

The following theorem provides a method for designing the set-membership filter that is used to compute the state estimation ellipsoid for the system (1)-(2) subject to the state equality constraint (3).

THEOREM 1 For the system (1)-(2) subject to the constraint (3), if the state $x_{k}$ belongs to its state estimation ellipsoid $\left(x_{k}-\hat{x}_{k}\right)^{T} P_{k}^{-1}\left(x_{k}-\hat{x}_{k}\right) \leq 1$, where $\hat{x}_{k}$ and $P_{k}>0$ are known, then one-step-ahead state $x_{k+1}$ resides in its state estimation ellipsoid $\left(x_{k+1}-\hat{x}_{k+1}\right)^{T} P_{k+1}^{-1}\left(x_{k+1}-\hat{x}_{k+1}\right) \leq 1$, if $P_{k+1}$ satisfies the following LMI

$$
\left[\begin{array}{ll}
-P_{k+1} & \Pi\left(\hat{x}_{k}, u_{k}\right) \\
\Pi\left(\hat{x}_{k}, u_{k}\right)^{T} & -\operatorname{diag}\left(1-\tau_{1}-\tau_{2}-\tau_{3}, \tau_{1} I, \tau_{2} Q_{k}^{-1}, \tau_{3} R_{k+1}^{-1}\right) \\
& \quad+N_{k}^{T} \Pi_{1}\left(\hat{x}_{k}\right)+\Pi_{1}\left(\hat{x}_{k}\right)^{T} N_{k}
\end{array}\right]<0
$$

by appropriately choosing $G_{k}, L_{k}, N_{k}, \tau_{1}>0, \tau_{2}>0$, $\tau_{3}>0$, and $\hat{x}_{k+1}$ is determined by

$$
\hat{x}_{k+1}=G_{k} \hat{x}_{k}+F_{k} u_{k}+L_{k} y_{k+1}
$$

where

$$
\begin{aligned}
\Pi\left(\hat{x}_{k}, u_{k}\right)=[ & \left(I-L_{k} C_{k+1}\right) A_{k} \hat{x}_{k}-G_{k} \hat{x}_{k}-L_{k} C_{k+1} F_{k} u_{k} \\
& \left(I-L_{k} C_{k+1}\right) A_{k} E_{k} \\
& \left.\left(I-L_{k} C_{k+1}\right) B_{k}-L_{k} D_{k+1}\right]
\end{aligned}
$$

and

$$
\Pi_{1}\left(\hat{x}_{k}\right)=\left[\begin{array}{llll}
S_{k} \hat{x}_{k}-s_{k} S_{k} E_{k} & 0 & 0
\end{array}\right] .
$$

PROOF If $\left(x_{k}-\hat{x}_{k}\right)^{T} P_{k}^{-1}\left(x_{k}-\hat{x}_{k}\right) \leq 1$, then there exists a $z$ with $\|z\| \leq 1$ such that

$$
x_{k}=\hat{x}_{k}+E_{k} z
$$

where $E_{k}$ is a factorisation of $P_{k}=E_{k} E_{k}^{T}$. Then one-step-ahead estimation error $x_{k+1}-\hat{x}_{k+1}$ is written as

$$
\begin{aligned}
x_{k+1} & -\hat{x}_{k+1} \\
& =A_{k} x_{k}+F_{k} u_{k}+B_{k} w_{k}-G_{k} \hat{x}_{k}-F_{k} u_{k}-L_{k} y_{k+1} .
\end{aligned}
$$

Substituting (2) into (18) yields

$$
\begin{aligned}
x_{k+1}- & \hat{x}_{k+1} \\
= & \left(I-L_{k} C_{k+1}\right) A_{k} x_{k}-G_{k} \hat{x}_{k}-L_{k} C_{k+1} F_{k} u_{k} \\
& +\left(I-L_{k} C_{k+1}\right) B_{k} w_{k}-L_{k} D_{k+1} v_{k+1} .
\end{aligned}
$$

By using (17), we have

$$
\begin{aligned}
x_{k+1}- & \hat{x}_{k+1} \\
= & \left(I-L_{k} C_{k+1}\right) A_{k} \hat{x}_{k}-G_{k} \hat{x}_{k}-L_{k} C_{k+1} F_{k} u_{k} \\
& +\left(I-L_{k} C_{k+1}\right) A_{k} E_{k} z+\left(I-L_{k} C_{k+1}\right) B_{k} w_{k}-L_{k} D_{k+1} v_{k+1} .
\end{aligned}
$$

Denoting

$$
\eta=\left[\begin{array}{c}
1 \\
z \\
w_{k} \\
v_{k+1}
\end{array}\right]
$$

we can rewrite (20) as follows:

$$
x_{k+1}-\hat{x}_{k+1}=\Pi\left(\hat{x}_{k}, u_{k}\right) \eta
$$

where $\Pi\left(\hat{x}_{k}, u_{k}\right)$ is defined in (15).

Hence, $\left(x_{k+1}-\hat{x}_{k+1}\right)^{T} P_{k+1}^{-1}\left(x_{k+1}-\hat{x}_{k+1}\right) \leq 1$ can be written as

$$
\eta^{T} \Pi\left(\hat{x}_{k}, u_{k}\right)^{T} P_{k+1}^{-1} \Pi\left(\hat{x}_{k}, u_{k}\right) \eta-\eta^{T} \operatorname{diag}(1,0,0,0) \eta<0 .
$$

Now $\|z\| \leq 1, w_{k}^{T} Q_{k}^{-1} w_{k} \leq 1$, and $v_{k+1}^{T} R_{k+1}^{-1} v_{k+1} \leq 1$ are also written in the following inequality form:

$$
\begin{array}{r}
\eta^{T} \operatorname{diag}(-1, I, 0,0) \eta \leq 0 \\
\eta^{T} \operatorname{diag}\left(-1,0, Q_{k}^{-1}, 0\right) \eta \leq 0 \\
\eta^{T} \operatorname{diag}\left(-1,0,0, R_{k+1}^{-1}\right) \eta \leq 0 .
\end{array}
$$

According to Lemma 1, the sufficient condition such that the inequalities (24)-(26) imply (23) to hold is that there exist positive scalars $\tau_{1}, \tau_{2}$, and $\tau_{3}$ such that

$$
\begin{gathered}
\Pi\left(\hat{x}_{k}, u_{k}\right)^{T} P_{k+1}^{-1} \Pi\left(\hat{x}_{k}, u_{k}\right)-\operatorname{diag}(1,0,0,0)-\tau_{1} \operatorname{diag}(-1, I, 0,0) \\
-\tau_{2} \operatorname{diag}\left(-1,0, Q_{k}^{-1}, 0\right)-\tau_{3} \operatorname{diag}\left(-1,0,0, R_{k+1}^{-1}\right) \leq 0 .
\end{gathered}
$$


Equation (27) is written in the following compact form:

$$
\begin{aligned}
& \Pi\left(\hat{x}_{k}, u_{k}\right)^{T} P_{k+1}^{-1} \Pi\left(\hat{x}_{k}, u_{k}\right) \\
& \quad-\operatorname{diag}\left(1-\tau_{1}-\tau_{2}-\tau_{3}, \tau_{1} I, \tau_{2} Q_{k}^{-1}, \tau_{3} R_{k+1}^{-1}\right) \leq 0 .
\end{aligned}
$$

Now we consider the state equality constraint (3). Substituting (17) into (3) yields

$$
S_{k} \hat{x}_{k}+S_{k} E_{k} z=s_{k} .
$$

Equation (29) can be expressed as

$$
\Pi_{1}\left(\hat{x}_{k}\right) \eta=0
$$

where $\Pi_{1}\left(\hat{x}_{k}\right)$ and $\eta$ are defined in (16) and (21), respectively.

We apply Finsler's Lemma 3 to (30) and (28). Then there exists an $N_{k}$ such that the following inequality holds:

$$
\begin{aligned}
& \Pi\left(\hat{x}_{k}, u_{k}\right)^{T} P_{k+1}^{-1} \Pi\left(\hat{x}_{k}, u_{k}\right) \\
& \quad-\operatorname{diag}\left(1-\tau_{1}-\tau_{2}-\tau_{3}, \tau_{1} I, \tau_{2} Q_{k}^{-1}, \tau_{3} R_{k+1}^{-1}\right) \\
& \quad+N_{k}^{T} \Pi_{1}\left(\hat{x}_{k}\right)+\Pi_{1}\left(\hat{x}_{k}\right)^{T} N_{k} \leq 0 .
\end{aligned}
$$

By using Schur complements in Lemma 2, (31) leads to (13). Thus, if $P_{k+1}$ satisfies LMI (13), and $\hat{x}_{k+1}$ is determined by (14), then one-step-ahead state $x_{k+1}$ resides in its state estimation ellipsoid $\left(x_{k+1}-\hat{x}_{k+1}\right)^{T} P_{k+1}^{-1}\left(x_{k+1}-\hat{x}_{k+1}\right) \leq 1$.

Theorem 1 outlines the principle of determining the current state estimation ellipsoid given the previous state estimation ellipsoid. However, it does not provide an optimal state estimation ellipsoid. Next, we apply the convex optimisation approach to determine an optimal ellipsoid. $P_{k+1}$ is obtained by solving the following optimisation problem:

$$
\min _{P_{k+1}>0, G_{k}, L_{k}, N_{k}, \tau_{1}>0, \tau_{2}>0, \tau_{3}>0} \operatorname{tr}\left(P_{k+1}\right)
$$

subject to (13)

and $\hat{x}_{k+1}$ is determined by (14), where $\Pi\left(\hat{x}_{k}, u_{k}\right)$ and $\Pi_{1}\left(\hat{x}_{k}\right)$ are defined in (15) and (16), respectively.

REMARK 3 We can see from Theorem 1 that the inequalities (13) are linear to the variables $P_{k+1}$, $G_{k}, L_{k}$, and $N_{k}, \tau_{1}, \tau_{2}, \tau_{3}$. Hence, the optimisation problems (32) can be solved by the existing semi-definite programming via interior-point approach $[4,12]$.

REMARK 4 The trace of $P_{k+1}$ is optimised at each time step in an effort to find the smallest ellipsoid for the state estimate. Other measures of the ellipsoid can also be introduced, for example, determinant $[8,13]$.

\section{SET-MEMBERSHIP FILTER DESIGN WITH STATE INEQUALITY CONSTRAINTS}

As a by-product in the previous section, we have developed the set-membership filter for the system (1)-(2) subject to the state inequality constraint (4). Due to the inequality constraint on the states, we can treat (4) as an additional inequality in Theorem 1 . The results are modified as follows.

THEOREM 2 For the system (1)-(2) subject to the constraint (4), if the state $x_{k}$ belongs to its state estimation ellipsoid $\left(x_{k}-\hat{x}_{k}\right)^{T} P_{k}^{-1}\left(x_{k}-\hat{x}_{k}\right) \leq 1$, where $\hat{x}_{k}$ and $P_{k}>0$ are known, then one-step-ahead state $x_{k+1}$ resides in its state estimation ellipsoid $\left(x_{k+1}-\hat{x}_{k+1}\right)^{T} P_{k+1}^{-1}\left(x_{k+1}-\hat{x}_{k+1}\right) \leq 1$, if $P_{k+1}$ satisfies the following LMI

$$
\left[\begin{array}{ll}
-P_{k+1} & \Pi\left(\hat{x}_{k}, u_{k}\right) \\
\Pi\left(\hat{x}_{k}, u_{k}\right)^{T} & -\operatorname{diag}\left(1-\tau_{1}-\tau_{2}-\tau_{3}-\tau_{4} a_{k}, \tau_{1} I, \tau_{2} Q_{k}^{-1}\right. \\
& \left.\tau_{3} R_{k+1}^{-1}\right)-\tau_{4} \Pi_{2}\left(\hat{x}_{k}\right)^{T} J_{k} \Pi_{2}\left(\hat{x}_{k}\right)
\end{array}\right] \leq 0
$$

by appropriately choosing $G_{k}, L_{k}, \tau_{1}>0, \tau_{2}>0$, $\tau_{3}>0, \tau_{4}>0$, and $\hat{x}_{k+1}$ is determined by

$$
\hat{x}_{k+1}=G_{k} \hat{x}_{k}+F_{k} u_{k}+L_{k} y_{k+1}
$$

where

$$
\Pi_{2}\left(\hat{x}_{k}\right)=\left[\begin{array}{llll}
\hat{x}_{k} & E_{k} & 0 & 0
\end{array}\right]
$$

and $\Pi\left(\hat{x}_{k}, u_{k}\right)$ is defined in (15).

PROOF According to the proof of Theorem 1, $\left(x_{k+1}-\hat{x}_{k+1}\right)^{T} P_{k+1}^{-1}\left(x_{k+1}-\hat{x}_{k+1}\right) \leq 1,\|z\| \leq 1$, $w_{k}^{T} Q_{k}^{-1} w_{k} \leq 1$, and $v_{k+1}^{T} R_{k+1}^{-1} v_{k+1} \leq 1$ can be expressed as (23), (24), (25), and (26), respectively.

Now we can rewrite (4) as

$$
\eta^{T} \Pi_{2}\left(\hat{x}_{k}\right)^{T} J_{k} \Pi_{2}\left(\hat{x}_{k}\right) \eta-\eta^{T} \operatorname{diag}\left(a_{k}, 0,0,0\right) \eta \leq 0
$$

where

$$
\Pi_{2}\left(\hat{x}_{k}\right)=\left[\begin{array}{llll}
\hat{x}_{k} & E_{k} & 0 & 0
\end{array}\right] .
$$

According to Lemma 1, the sufficient condition such that the inequalities (24)-(26), (36) imply (23) to hold is that there exist positive scalars $\tau_{1}, \tau_{2}, \tau_{3}$, and $\tau_{4}$ such that

$$
\begin{gathered}
\Pi\left(\hat{x}_{k}, u_{k}\right)^{T} P_{k+1}^{-1} \Pi\left(\hat{x}_{k}, u_{k}\right)-\operatorname{diag}(1,0,0,0)-\tau_{1} \operatorname{diag}(-1, I, 0,0) \\
\quad-\tau_{2} \operatorname{diag}\left(-1,0, Q_{k}^{-1}, 0\right)-\tau_{3} \operatorname{diag}\left(-1,0,0, R_{k+1}^{-1}\right) \\
-\tau_{4}\left[\Pi_{2}\left(\hat{x}_{k}\right)^{T} J_{k} \Pi_{2}\left(\hat{x}_{k}\right)-\operatorname{diag}\left(a_{k}, 0,0,0\right)\right] \leq 0 .
\end{gathered}
$$

Equation (38) is written in the following compact form:

$$
\begin{aligned}
& \Pi\left(\hat{x}_{k}, u_{k}\right)^{T} P_{k+1}^{-1} \Pi\left(\hat{x}_{k}, u_{k}\right) \\
& \quad-\operatorname{diag}\left(1-\tau_{1}-\tau_{2}-\tau_{3}-\tau_{4} a_{k}, \tau_{1} I, \tau_{2} Q_{k}^{-1}, \tau_{3} R_{k+1}^{-1}\right) \\
& \quad-\tau_{4} \Pi_{2}\left(\hat{x}_{k}\right)^{T} J_{k} \Pi_{2}\left(\hat{x}_{k}\right) \leq 0 .
\end{aligned}
$$




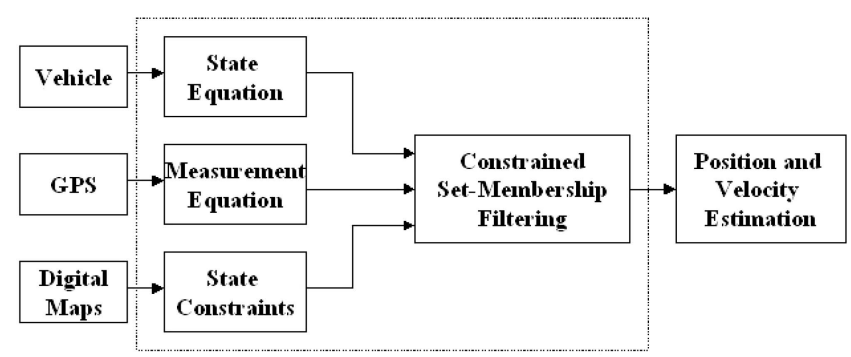

Fig. 1. Block diagram of implementation of vehicle position and velocity estimation by constrained set-membership filter.

By using Schur complements in Lemma 2, (39) leads to (33). Thus, if $P_{k+1}$ satisfies LMI (33), and $\hat{x}_{k+1}$ is determined by (34), then one-step-ahead state $x_{k+1}$ resides in its state estimation ellipsoid $\left(x_{k+1}-\hat{x}_{k+1}\right)^{T} P_{k+1}^{-1}\left(x_{k+1}-\hat{x}_{k+1}\right) \leq 1$.

According to Theorem 2, we can apply the optimisation method to determine $P_{k+1}$. The optimisation problem is cast as follows:

$$
\min _{P_{k+1}>0, G_{k}, L_{k}, \tau_{1}>0, \tau_{2}>0, \tau_{3}>0, \tau_{4}>0} \operatorname{tr}\left(P_{k+1}\right)
$$

subject to (33)

and $\hat{x}_{k+1}$ is determined by (34), where $\Pi\left(\hat{x}_{k}, u_{k}\right)$ and $\Pi_{2}\left(\hat{x}_{k}\right)$ are defined in (15) and (35), respectively.

\section{A VEHICLE TRACKING EXAMPLE}

In this section, we consider a digital-map-based vehicle tracking system. The system consists of a moving vehicle, a Global Positioning System (GPS) receiver and a digital map database that contains the road geometry information. Our purpose is to design a constrained set-membership filter to estimate the position and velocity of the vehicle. The block diagram is shown in Fig. 1.

The vehicle dynamics are described by the following equation $[23,24]$

$$
x_{k+1}=\left[\begin{array}{llll}
1 & 0 & T & 0 \\
0 & 1 & 0 & T \\
0 & 0 & 1 & 0 \\
0 & 0 & 0 & 1
\end{array}\right] x_{k}+\left[\begin{array}{c}
0 \\
0 \\
0.4 T \sin \theta \\
T \cos \theta
\end{array}\right] u_{k}+\left[\begin{array}{l}
2 \\
1 \\
1 \\
1
\end{array}\right] w_{k}
$$

where the first two components of $x_{k}$ are $\mathrm{x}$ - and $\mathrm{y}$-axis positions, denoted by $x$ and $y$, respectively; the last two components of $x_{k}$ are $\mathrm{x}$ - and $\mathrm{y}$-axis velocities, denoted by $v_{x}$ and $v_{y}$, respectively; $u_{k}$ is the commanded acceleration; $w_{k}$ represents process disturbances due to potholes and the like which may be non-Gaussian but belongs to a specified ellipsoidal set; $T$ is the sample period; and $\theta$ is the road orientation angle from the $\mathrm{x}$-axis.

The GPS measurement equation can be written as

$$
y_{k}=\left[\begin{array}{llll}
1 & 0 & 0 & 0 \\
0 & 1 & 0 & 0
\end{array}\right] x_{k}+\left[\begin{array}{l}
1 \\
2
\end{array}\right] v_{k}
$$

where $y_{k}$ is the GPS measurement; and $v_{k}$ is the GPS measurement noise.

Using the road direction obtained from a digital map database, some constraints between the vehicle states can be obtained. For instance, if it is known that the vehicle is travelling on a straight road with a heading of $\theta$, then the matrix $S_{k}$ and the vector $s_{k}$ of (3) can be given as follows:

$$
S_{k}=\left[\begin{array}{cccc}
1 & -\tan \theta & 0 & 0 \\
0 & 0 & 1 & -\tan \theta
\end{array}\right], \quad s_{k}=\left[\begin{array}{ll}
0 & 0
\end{array}\right]^{T} .
$$

In the simulation, the sample period $T$ is chosen as $3 \mathrm{~s}$ and the road orientation angle is set to a constant $60^{\circ}$. The commanded acceleration $u_{k}$ is alternately set to $\pm 1 \mathrm{~m} / \mathrm{s}^{2}$, as if the vehicle was alternately accelerating and decelerating in traffic. $w_{k}$ and $v_{k}$ is assumed as $0.5 \sin (2 k)$ and $0.5 \sin (30 k)$, respectively. The initial state is set as $x_{0}=\left[\begin{array}{llll}5 & 5 \sqrt{3} & 2 & 2\end{array} \sqrt{3}^{T}\right.$, which belongs to the ellipsoid $\mathcal{E}\left(P_{0}, \hat{x}_{0}\right)=\left\{x_{0}\right.$ : $\left(x_{0}-\hat{x}_{0}\right)^{T} P_{0}^{-1}\left(x_{0}-\hat{x}_{0}\right) \leq 1$, where $\hat{x}_{0}=\left[\begin{array}{llll}0 & 7 & 0 & 3\end{array}\right]^{T}$, and

$$
P_{0}=\left[\begin{array}{cccc}
100 & 0 & 0 & 0 \\
0 & 100 & 0 & 0 \\
0 & 0 & 10 & 0 \\
0 & 0 & 0 & 10
\end{array}\right]
$$

For all $k, Q_{k}=1$ and $R_{k}=1$.

The simulation results are obtained by solving the semi-definite programming problem (32) under Matlab 6.5 with YALMIP 3.0 and SeDuMi 1.1 [12]. Figs. 2 and 3 show that the actual vehicle positions and the estimates of the vehicle positions along $\mathrm{x}$ - and $y$-axis by using the constrained set-membership filter. Fig. 4 provides the actual position of the vehicle on plane and shows that the actual travelling direction of the vehicle is almost $60^{\circ}$ from the $\mathrm{x}$-axis. Figs. 5 and 6 show the position estimates and their upper bounds and lower bounds relative to the actual positions. The results confirm that the actual vehicle positions always reside between the upper bounds and lower bounds of its estimates. Therefore, the vehicle belongs to the estimated region at any time step. We can conclude that the target is fully tracked by using the constrained set-membership filter. Moreover, we can see from Figs. 7-8 that the actual vehicle velocities also reside between the upper bounds and lower bounds of its estimates. Therefore, we can monitor the maximum speed of the vehicle from the GPS vehicle position measurement. We can also see from Figs. 7-8 that the lower bounds become negative sometimes. However, that does not mean that the true velocity is negative. This only means that the true velocity belongs between the negative and positive velocities. We do not know the true velocity exactly (negative or positive velocities, i.e., forward or backward). Fig. 9 shows $\left(x_{k}-\hat{x}_{k}\right)^{T} P_{k}^{-1}\left(x_{k}-\hat{x}_{k}\right)$ as a function of $k$ and confirms that the design performance (9) is satisfied. 


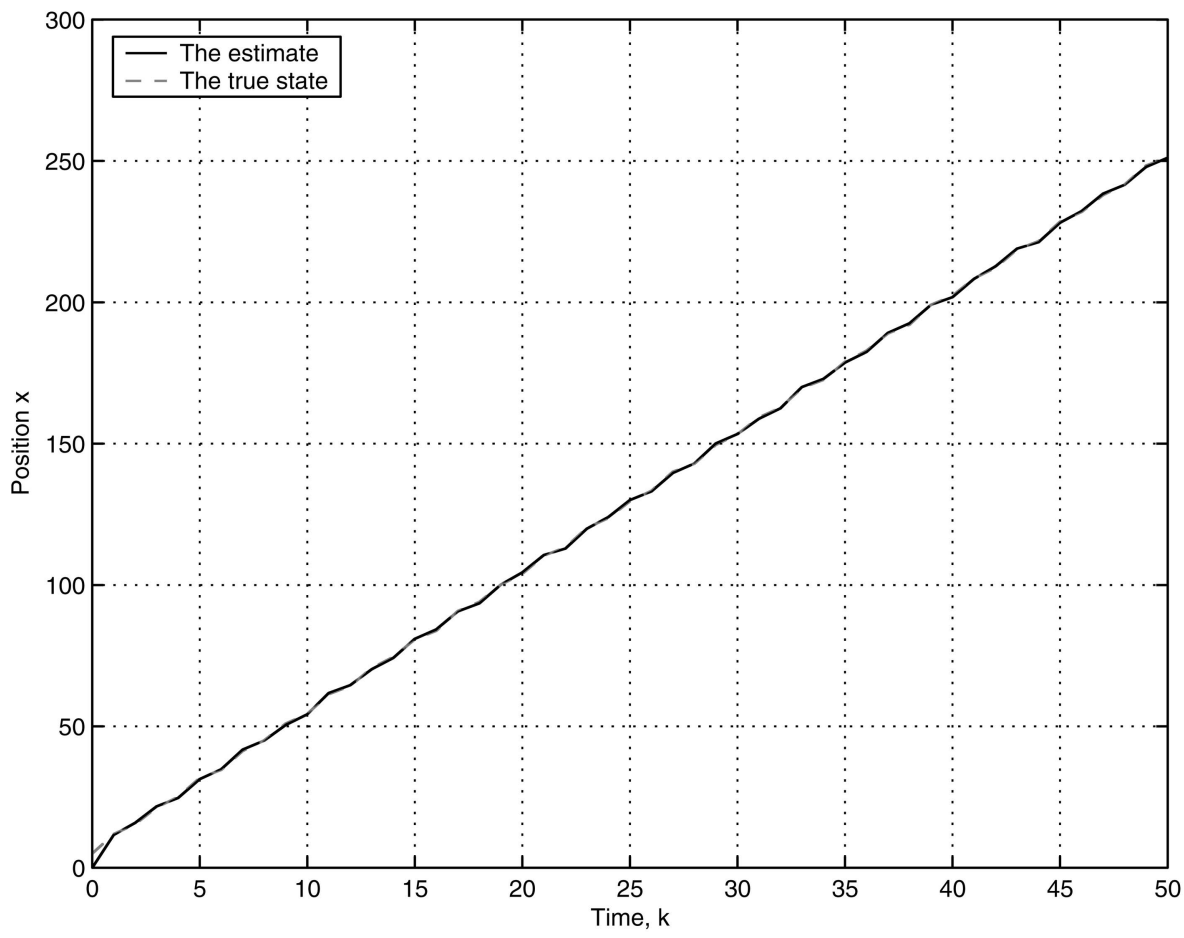

Fig. 2. True value and its estimate for vehicle position with proposed filter.

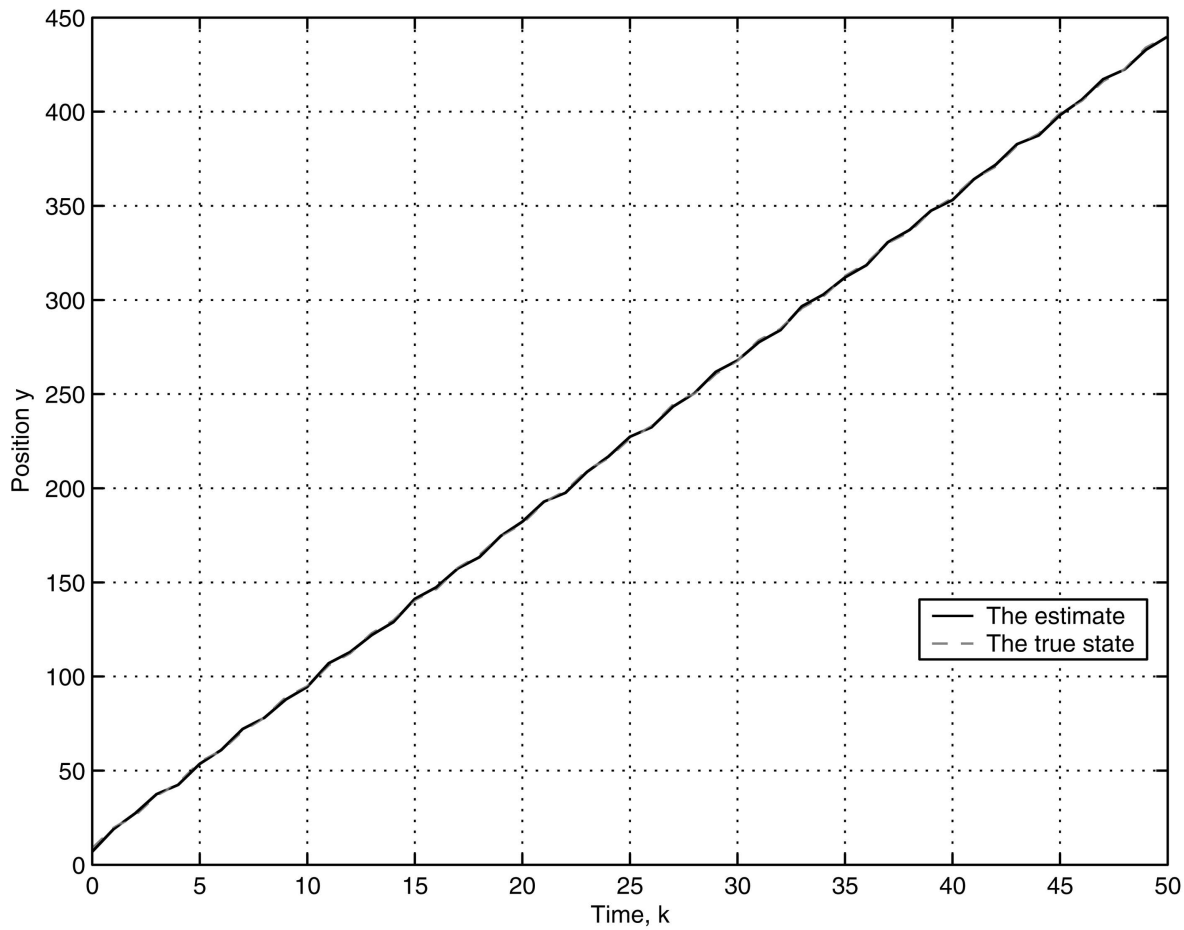

Fig. 3. True value and its estimate for vehicle position with proposed filter.

We also conducted the simulations under the state and measurement noises as normal distribution, uniform distribution, and outliers. Their maximum values are below the above bounds in all the process and measurement noises. The simulation results show that the different noises produce slight different trajectories of the true states. However, whatever the true states change, they always reside between the upper bounds and lower bounds of their estimates. The upper and lower bounds of their estimates depend on the bounds of noises and not noises themselves. Due to too many similar figures, they are omitted.

For comparison purposes, we use the set-membership filtering proposed in [13] to track the 


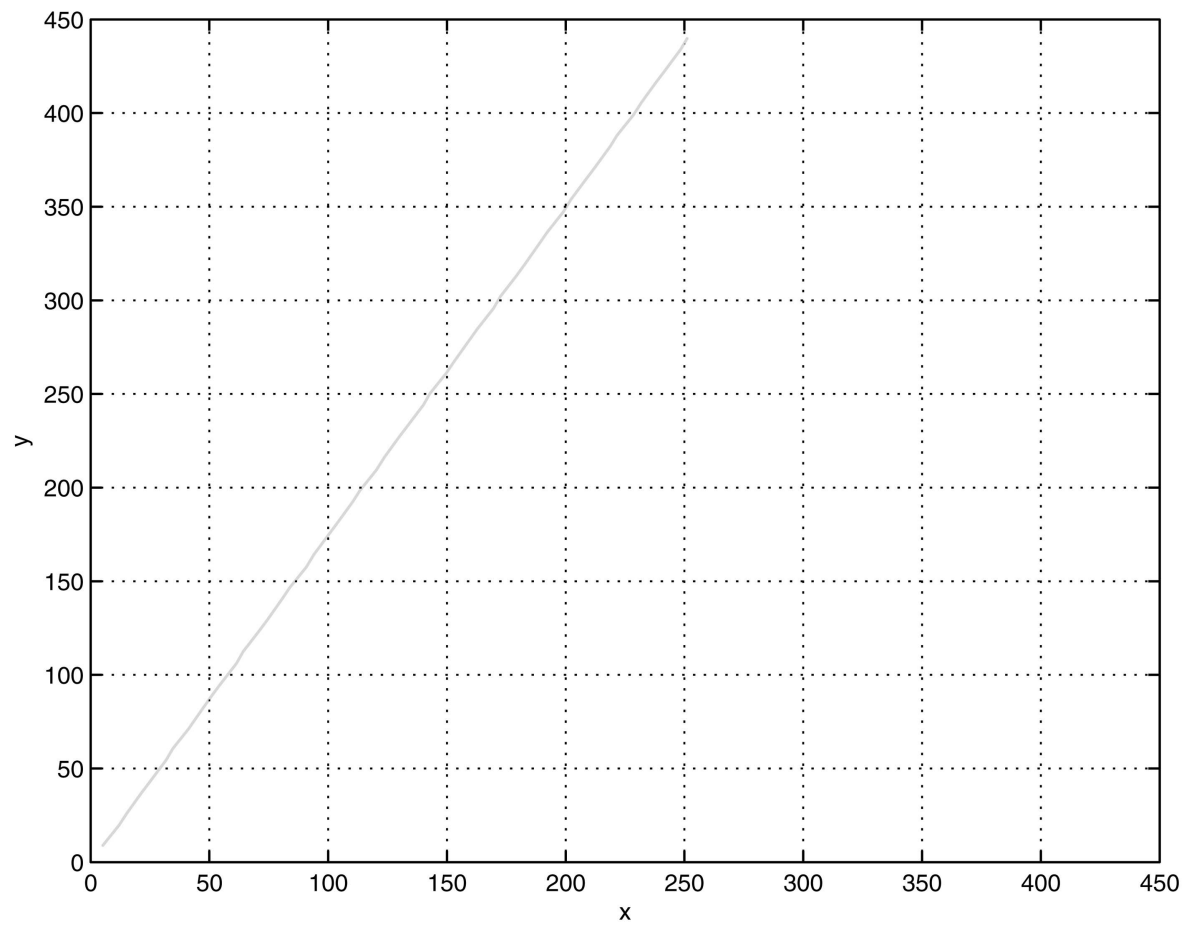

Fig. 4. Relationship between $x$ and $y$ in true state values.

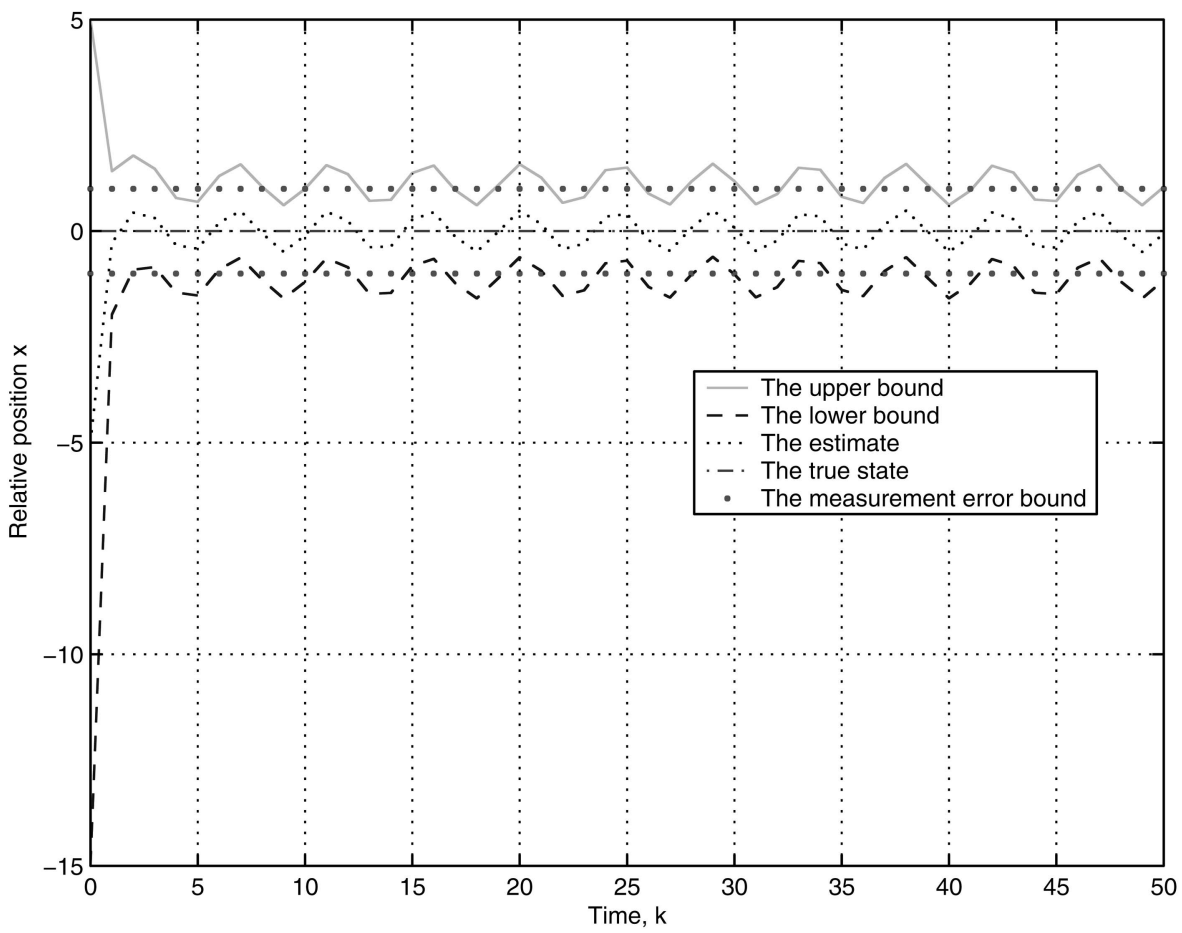

Fig. 5. Upper bound, lower bound, and estimate relative to true value for vehicle position with proposed filter and measurement error bound.

above vehicle, where the state constraint is regarded as a fictitious measurement. The same conditions are applied in this simulation. The comparison of the trace of $P_{k+1}$ between the proposed algorithm and the algorithm in [13] is depicted in Fig. 10. We can see from Fig. 10 that the trace of $P_{k+1}$ of our algorithm appears in a large value at the beginning (dynamic process), but after that the trace of $P_{k+1}$ of our algorithm is much smaller than that of the algorithm in [13]. This means that our algorithm is less conservative than the algorithm in [13]. We therefore can provide the tight bounds and 


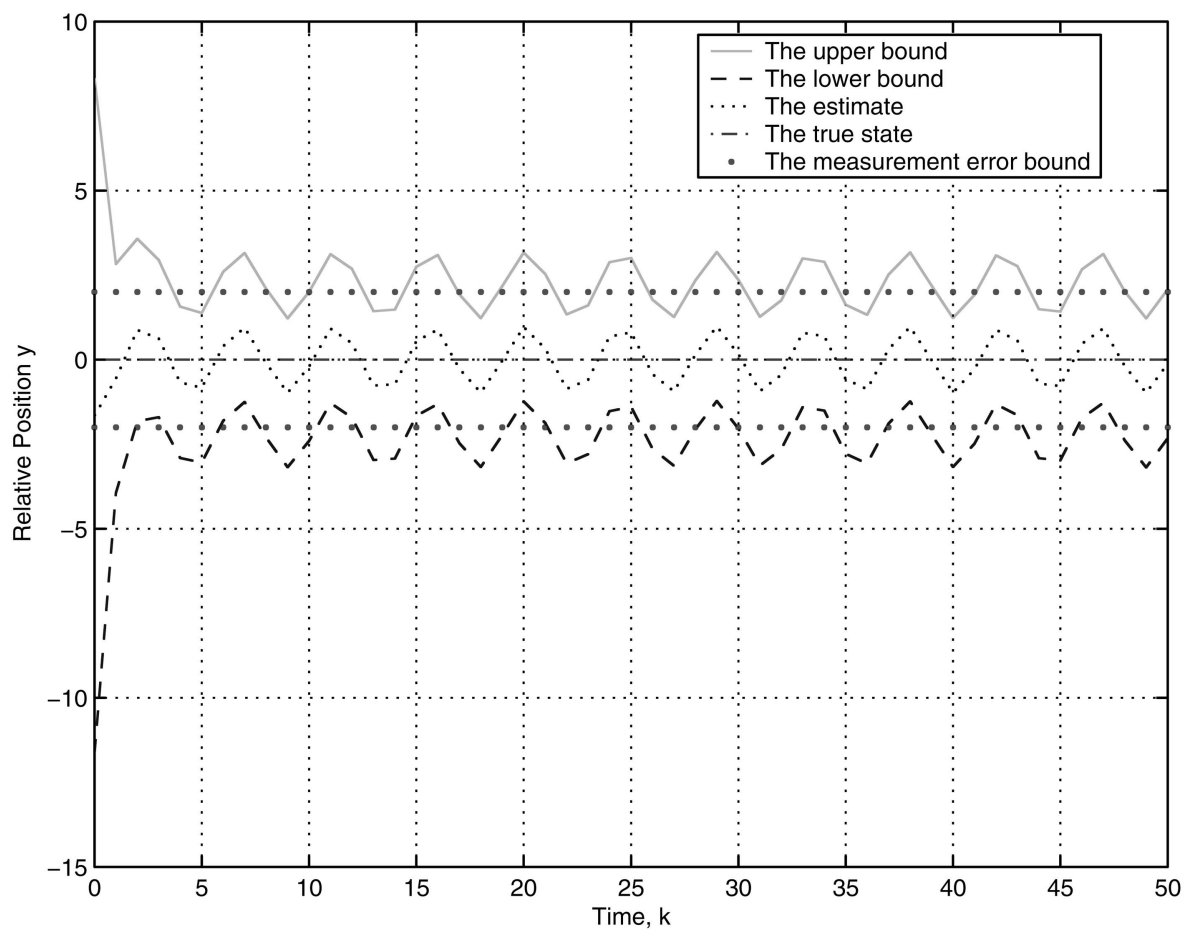

Fig. 6. Upper bound, lower bound, and estimate relative to true value for vehicle position with proposed filter and measurement error bound.

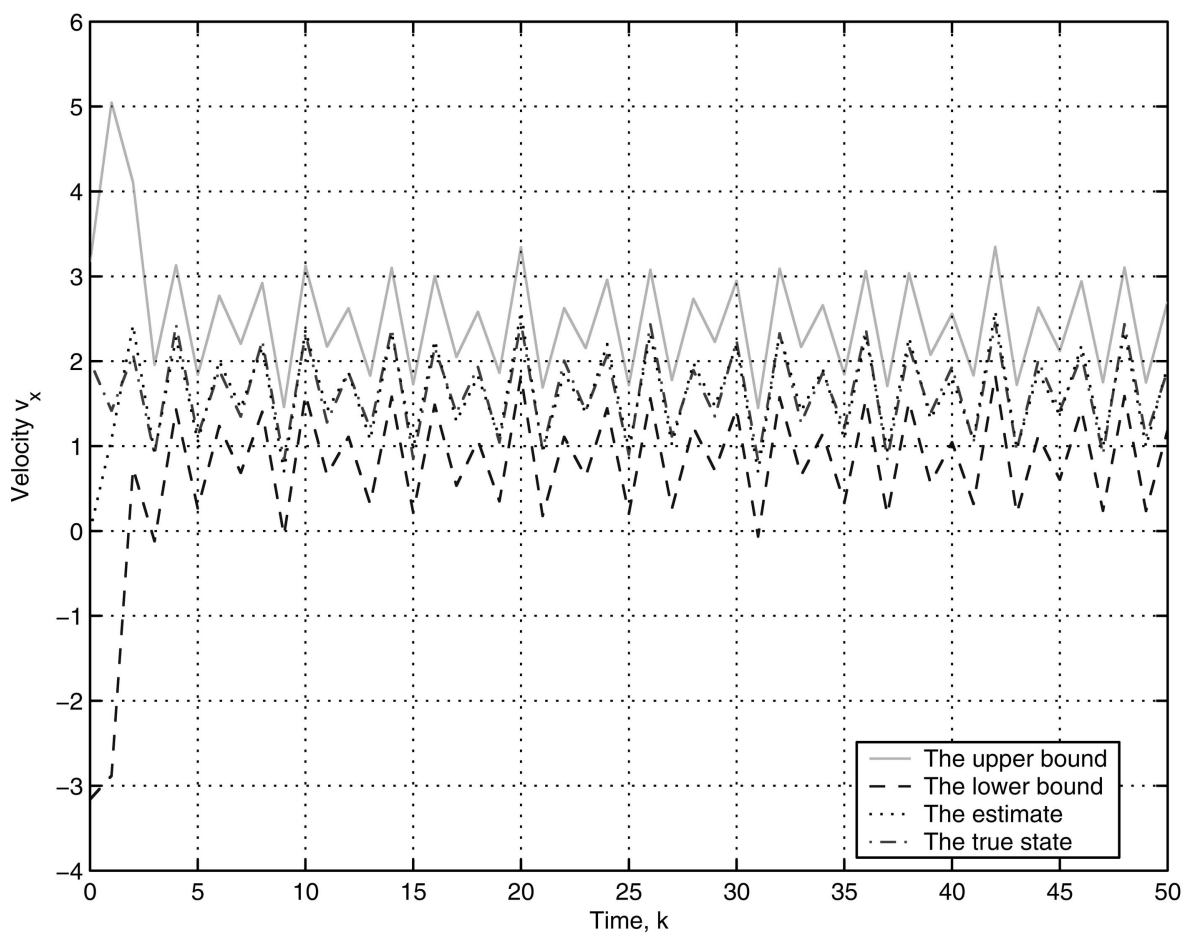

Fig. 7. True state value, its estimate and bounds for vehicle velocity with proposed filter.

locate the vehicle more accurately. However, the computation of our algorithm is more intensive than the algorithm proposed in [13], because at every step the optimization problem (32) is solved by numerical iterative algorithm using semi-definite programming. In this example, the algorithm in [13] only needs $2 \mathrm{~s}$ of CPU time to run, whereas our algorithm needs $27 \mathrm{~s}$ of CPU time.

\section{CONCLUSIONS}

This paper has considered the set-membership filtering problem for discrete-time systems with 


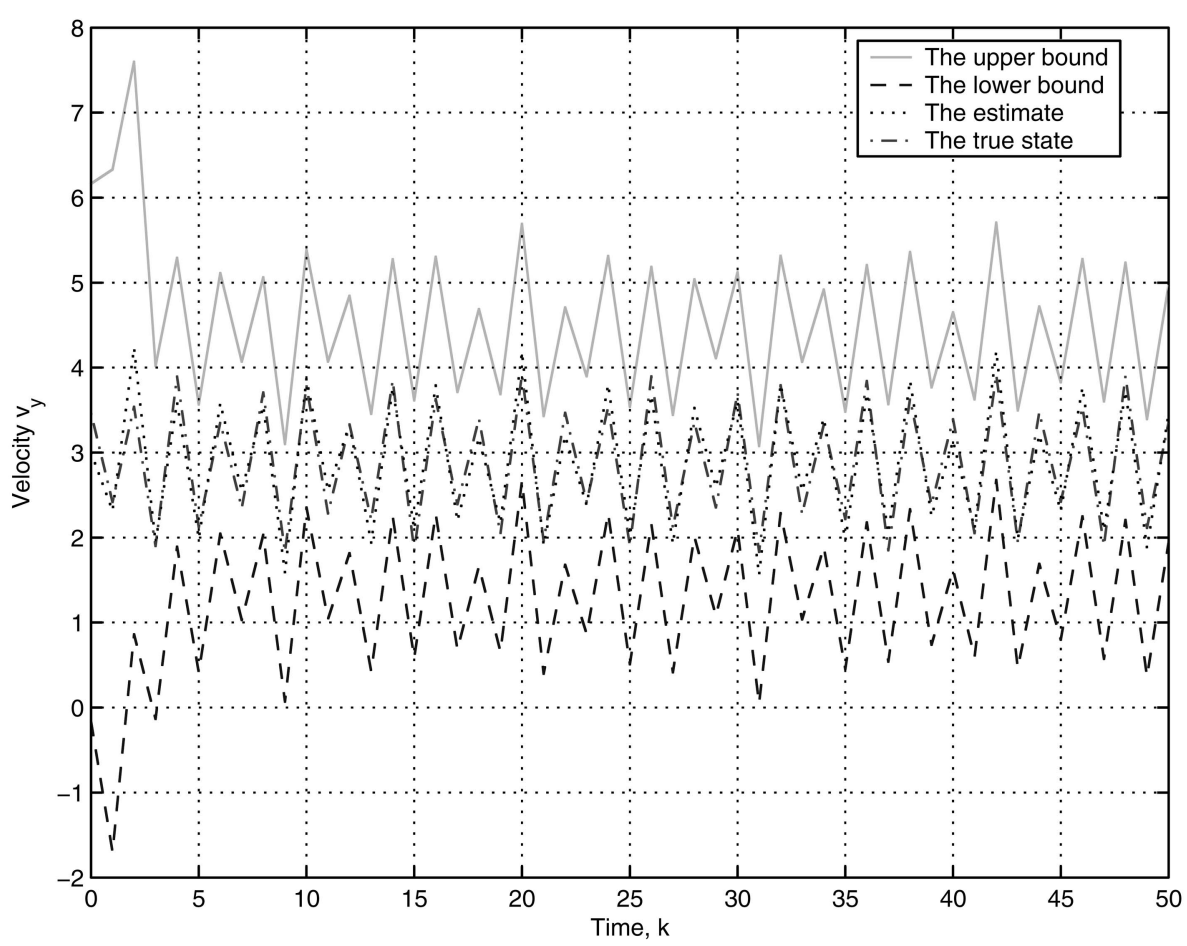

Fig. 8. True state value, its estimate and bounds for vehicle velocity with proposed filter.

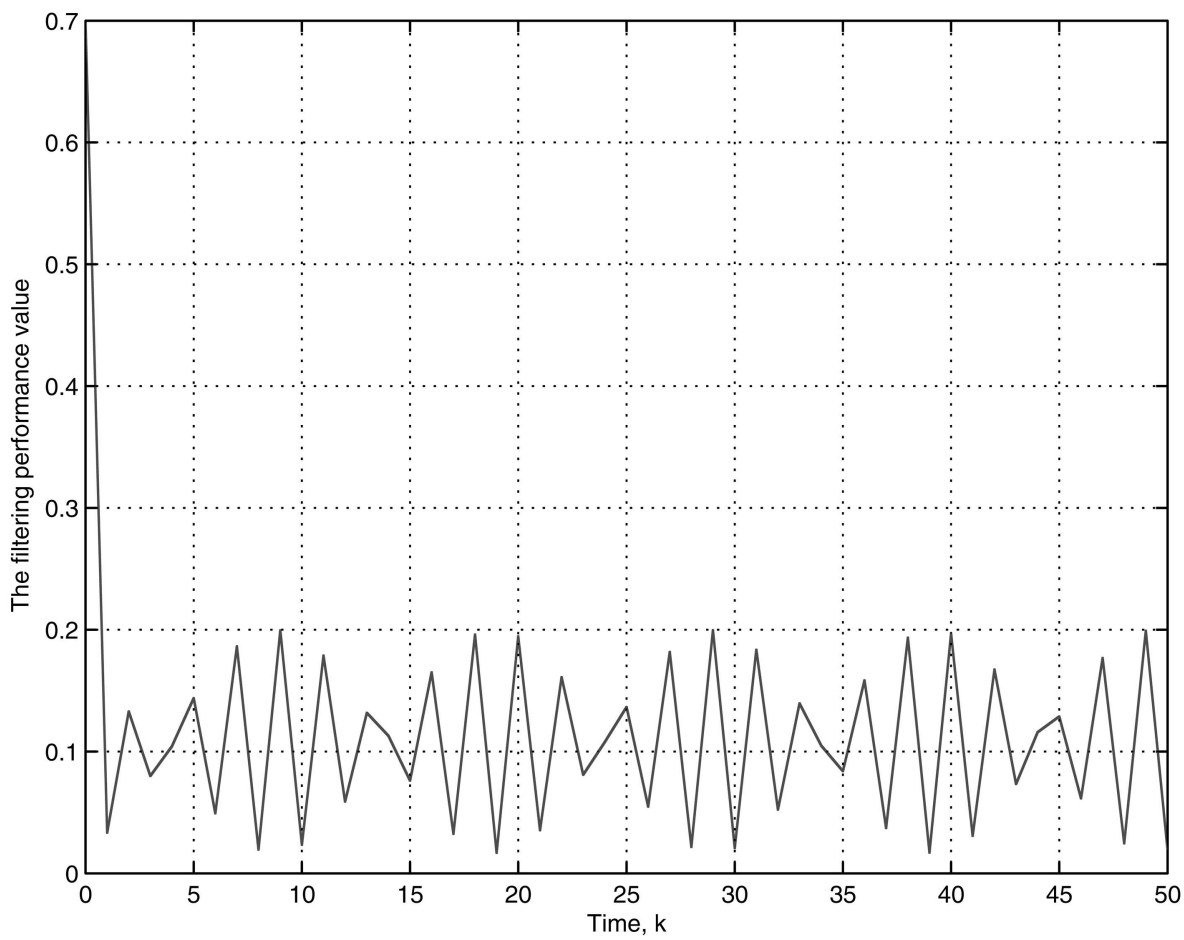

Fig. 9. Filtering performance $\left(x_{k}-\hat{x}_{k}\right)^{T} P_{k}^{-1}\left(x_{k}-\hat{x}_{k}\right)$.

equality and inequality constraints between their state variables. We have formulated the set-membership filtering problem as finding the set of estimates that belongs to an ellipsoid. A centre and a shape matrix of the ellipsoid have been introduced to represent the set of estimates so that the S-procedure can be used to handle several constraints in the problem. We finally applied Finsler's Lemma to project the set of estimates onto the constrained surface. The solution has been obtained by solving a set of LMIs recursively. A vehicle tracking example has demonstrated the feasibility of the proposed set-membership filtering with state equality constraints. However, in practical application, it is hard to guarantee that the state 


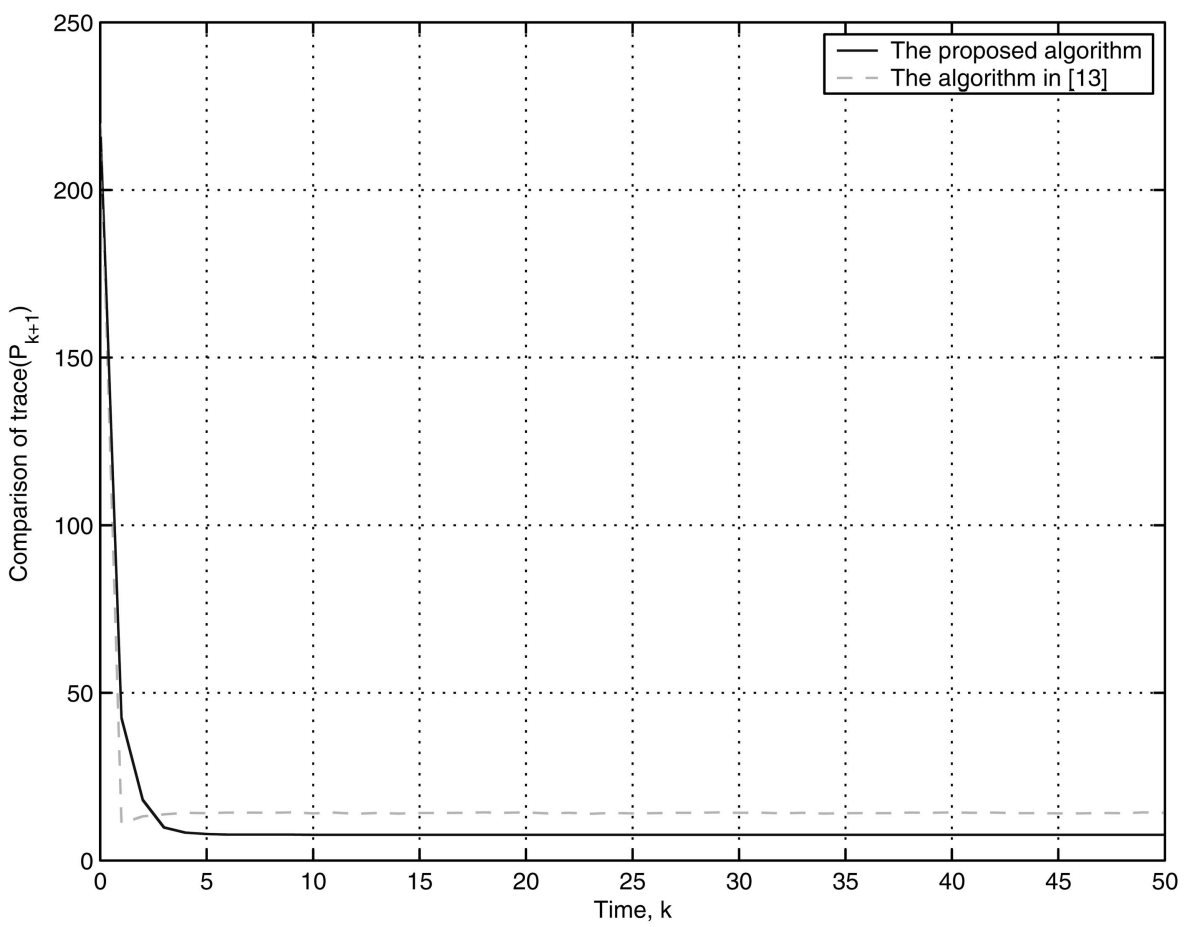

Fig. 10. Comparison of trace of $P_{k+1}$ between proposed algorithm and algorithm in [13].

equality constraint is exactly satisfied due to non-zero process noise. A small error should be taken into account in design. This will be one of our future research topics. The much more challenging research topic is how to optimise the upper bounds and lower bounds according to the measurement error bounds, and further reduce the conservatism of the possible estimation sets. Our method can also be extended to nonlinear state constraints and nonlinear dynamics systems.

\section{FUWEN YANG ${ }^{1}$ \\ YONGMIN LI \\ Dept. of Information Systems and Computing \\ Brunel University \\ Uxbridge, Middlesex, UB8 3PH \\ United Kingdom \\ E-mail: (fwyang@ecust.edu.cn) \\ ${ }^{1}$ Current address: \\ Dept. of Automation \\ East China University of Science and Technology Automation \\ 130 Meilong Road \\ Shanghai, 200237 \\ China}

\section{REFERENCES}

[1] Alouani, A. T., and Blair, W. D.

Use of a kinematic constraint in tracking constant speed, maneuvering targets.

IEEE Transactions on Automatic Control, 38, 7 (July

1993), 1107-1111.

[2] Anderson, B. D. O., and Moore, J. B.

Optimal Filtering.

Englewood Cliffs, NJ: Prentice-Hall, 1979.
[3] Bertsekas, D. P., and Rhodes, I. B.

Recursive state estimation for a set-membership description of uncertainty.

IEEE Transactions on Automatic Control, AC-16, 2 (Apr. 1971), 117-128.

[4] Boyd, S., Ghaoui, L. E., Feron, E., and Balakrishnan, V. Linear Matrix Inequalities in System and Control Theory (SIAM Studies in Applied Mathematics).

SIAM, Philadelphia, PA, 1994.

[5] Doran, H. E.

Constraining Kalman filter and smoothing estimates to satisfy time-varying restrictions.

The Review of Economics and Statistics, 74, 3 (1992), 568-572.

[6] Durieu, C., Walter, E., and Polyak, B.

Multi-input multi-output ellipsoidal state bounding. Journal of Optimization Theory and Applications, 111, 2 (Nov. 2001), 273-303.

[7] Geeter, J. D., Brussel, H. V., and De Schutter, J. A smoothly constrained Kalman filter. IEEE Transactions on Pattern Analysis and Machine Intelligence, 19, 10 (Oct. 1997), 1171-1177.

[8] El Ghaoui, L., and Calafiore, G.

Robust filtering for discrete-time systems with bounded noise and parametric uncertainty.

IEEE Transactions on Automatic Control, 46, 7 (July 2001), 1084-1089.

[9] Julier, S. J., and LaViola, J. J., Jr. On Kalman filtering with nonlinear equality constraints. IEEE Transactions on Signal Processing, 55, 6 (June 2007), 2774-2784.

[10] Kurzhanski, A. B., and Valyi, I. Ellipsoidal Calculus for Estimation and Control. Boston: Birkhauser, 1996.

[11] Li, W., and Leung, $\mathrm{H}$. Constrained unscented Kalman filter based fusion of GPS/INS/digital map for vehicle localization. In Proceedings of IEEE Intelligent Transportation Systems, Oct. 2003, 1362-1367. 
YALMIP: A toolbox for modeling and optimization in Matlab.

In Proceedings of the IEEE CACSD Symposium, Taipei, Taiwan, 2004.

[13] Maskarov, D. G., and Norton, J. P.

State bounding with ellipsoidal set description of the uncertainty.

International Journal of Control, 65, 5 (1996), 847-866.

[14] Morrell, D. R., and Stirling, W. C.

Set-valued filtering and smoothing.

IEEE Transactions on Systems, Man and Cybernetics, 21, 1 (Jan. 1991), 184-193.

[15] Mourikis, A. I., and Roumeliotis, S. I.

A multi-state constraint Kalman filter for vision-aided inertial navigation.

In Proceedings of the International Conference on Robotics and Automation, Italy, Apr. 2007, 3565-3572.

[16] Polyak, B., Nazin, S., Durieu, C., and Walter, E.

Ellipsoidal estimation under model uncertainty.

Presented at the 15th Triennial World Congress,

Barcelona, Spain, July 2002.

[17] Ra, W. S., Jin, S. H., and Park, J. B.

Set-valued estimation approach to recursive robust $H_{\infty}$ filtering.

IEE Proceedings on Control Theory Applications, 151, 6 (June 2004), 773-782.

[18] Savkin, A. V., and Petersen, I. R.

Recursive state estimation for uncertain systems with an integral quadratic constraint

IEEE Transactions on Automatic Control, 40, 6 (June 1995), 1080-1083.

[19] Savkin, A. V., and Petersen, I. R.

Robust state estimation and model validation for discrete-time uncertain systems with a deterministic description of noise and uncertainty. Automatica, 34, 2 (1998), 271-274.

[20] Schweppe, F. C.

Recursive state estimation: Unknown but vounded errors and system inputs.

IEEE Transactions on Automatic Control, AC-13, 1 (Feb. 1968), 22-28.

[21] Shamma, J. S., and Tu, K-Y.

Set-valued observers and optimal disturbance rejection. IEEE Transactions on Automatic Control, 44, 2 (Feb. 1999), 253-264.

[22] Shen, S., Hong, L., and Cong, S.

Reliable road vehicle collision prediction with constrained filter.

Signal Processing, 86, 3 (2006), 3339-3356.

[23] Simon, D.

A game theory approach to constrained minimax state estimation.

IEEE Transactions on Signal Processing, 54, 2 (Feb. 2006), 405-412.

[24] Simon, D., and Chia, T.

Kalman filtering with state equality constraints.

IEEE Transactions on Aerospace and Electronic Systems,

38, 1 (Jan. 2002), 128-136.

[25] Skelton, R. E., Iwasaki, T., and Grigoriadis, K. A Unified Algebraic Approach to Linear Control Design. Bristol, PA: Taylor \& Francis, 1998.

[26] Tahk, M., and Speyer, J. L.

Target tracking problems subject o kinematic constraints. IEEE Transactions on Automatic Control, 35, 3 (Mar.

1990), 324-326.
[27] Wang, Z., Yang, F., Ho, D. W. C., and Liu, X.

Robust finite-horizon filtering for stochastic systems with missing measurements.

IEEE Signal Processing Letters, 12, 6 (2005), 437-440.

[28] Wang, Z., Yang, D., Ho, D. W. C., and Liu, X.

Robust $H_{\infty}$ filtering for stochastic time-delay systems with missing measurements.

IEEE Transactions on Signal Processing, 54, 7 (July 2006), 2579-2587.

[29] Witsenhausen, H. S.

Sets of possible states of linear systems given perturbed observations.

IEEE Transactions on Automatic Control, AC-13, 5 (May 1968), 556-558.

[30] Xie, L., Lu, L., Zhang, D., and Zhang, H.

Improved robust $H_{2}$ and $H_{\infty}$ filter for uncertain discrete-time systems.

Automatica, 40 (2004), 873-880.

[31] Yang, C., and Blasch, E.

Kalman filtering with nonlinear state constraints. In Proceedings of the 9th International Conference on Information Fusion, Italy, July 2006, 1-8.

[32] Yang, F., and Hung, Y. S.

Robust mixed $H_{2} / H_{\infty}$ filtering with regional pole assignment for uncertain discrete-time systems.

IEEE Transactions on Circuits and Systems-I, 49, 8 (Aug. 2002), 1236-1241.

[33] Yang, F., Wang, Z., and Hung, Y. S.

Robust Kalman filtering for discrete time-varying uncertain systems with multiplicative noise.

IEEE Transactions on Automatic Control, 47, 7 (July 2002), 1179-1183.

[34] Yang, F., Wang, Z., Ho, D. W. C., and Liu, X.

Robust $\mathrm{H}_{2}$ filtering for a class of systems with stochastic nonlinearities.

IEEE Transactions on Circuits and Systems-II, 53, 3 (Mar. 2006), 235-239.

[35] de Oliveira, M.

Linear Control Design: Linear Control and Filter Design. Lecture note, $2008 \mathrm{http}: / /$ maecourses.ucsd.edu/mae280b/ Lecture\%20Set\%20D/design_1.pdf.

[36] Sinha, A., Kirubarajan, T., and Bar-Shalom, Y

Application of the Kalman-Levy filter for tracking maneuvering targets.

IEEE Transactions on Aerospace and Electronic Systems, 43, 3 (July 2007), 1099-1107.

[37] Cheng, Y., and Singh, T.

Efficient particle filtering for road-constrained target tracking.

IEEE Transactions on Aerospace and Electronic Systems, 43, 4 (Oct. 2007), 1454-1469.

[38] Chen, G., Wang, J., and Shieh, L. S.

Interval Kalman filtering.

IEEE Transactions on Aerospace and Electronic Systems, 33, 1 (Jan. 1997), 250-259. 